\title{
Improvement of Linear Amplification with Nonlinear Components Based on COX's Theory
}

\author{
Chengguo Liu, Shang-he Liu \\ Electrostatic \& Electromagnetic Protection Institute \\ Ordnance Engineering College, Shijiazhuang, China \\ Shijiazhuang, China \\ e-mail: liucg@whut.edu.cn
}

\author{
Chengguo Liu, Guowei Yang, Zhi Peng Wu \\ RF \&Microwave Technology Research Center \\ Wuhan University of Technology \\ Wuhan, China \\ e-mail: seamollon@163.com
}

\begin{abstract}
A theoretical research on Linear Amplification with Nonlinear Components (LINC) is described with an improved theory to COX's proposed in this article. Input RF signal is separated into envelope component and constant envelope angle-modulated component in the circuit. Two branches of constant envelope angle-modulated component phase modulated by envelope component respectively and conversely are converted frequency and amplified. At last, the amplified up-convertion input signal is achieved through a subtracting circuit. Because the amplification of constant envelope signal can be carried on without distortion in nonlinear region, the whole work efficiency is very high. In this article the power gain of LINC amplifier is derived as and the simulation of the circuit is operated and analyzed. The expectation of theoretical amplification effect is realized and high power efficiency is obtained.
\end{abstract}

Keywords- RF power amplifiers; Microwave Circuit; power efficiency

\section{INTRODUCTION}

RF power amplifiers are core components in modern communication system of which efficiency and linearity have great influence on the whole system performance, while they are a pair of contradictory index, and demand for tradeoffs[1]. With the development of modern communication techniques, spectrum resources are more and more intense[2], and the amount of information transmission is more and more large. So there are increasingly complicated signal modulation techniques emerging as the state-of- the-art technique required, such as multi-carrier technique, orthogonal frequency division multiplexing, Multivariate linear amplitude modulation technology etc.[3]. Their productions are modulated signals that have unconstant envelopes and large PAPR[4]. As a result, the signals are prone to working in the nonlinear area of PAs which leading to distortion and apparent spectral expansion interference. That's why a good performance of linearity is needed in PAs. Because PAs have the largest power consuming in the whole communication system, performance of high efficiency is an inevitable requirement.

In LINC technique[6][7], PAs can operate in saturated state and therefore have high efficiency, then a good performance of linearity can be achieved as long as keeping the two branches balance of amplitude and phase. Hence, this technique is now widely used in RFPA circuits[8]-[11]. In this article, the basic principle of LINC is studied and some valid improvements are raised with simulation analysis followed.

\section{FUNDAMENTALS}

For a general band-pass signal

$$
S(t)=E(t) \cos \left[\omega_{0} t+\theta(t)\right]
$$

where $E(t)=E_{m} \sin \phi(t) \geq 0$ is a real envelope. It is looked as input signal to a LINC amplifier and input into component separator. As is shown in Figure 1.

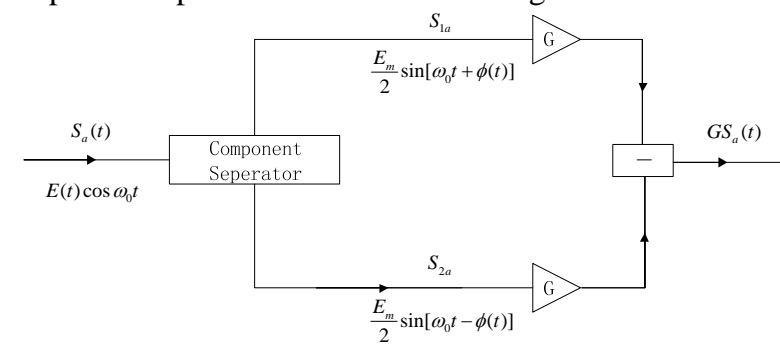

Figure 1. Schematic of LINC amplifier.

After processed by component separator there are two constant amplitude component signals $S_{1}(t)$ and $S_{2}(t)$ :

$$
\begin{aligned}
& S_{1}(t)=\frac{E_{m}}{2} \sin \left[\omega_{0} t+\theta(t)+\phi(t)\right] \\
& S_{2}(t)=\frac{E_{m}}{2} \sin \left[\omega_{0} t+\theta(t)-\phi(t)\right]
\end{aligned}
$$

The two amplifiers shown in Figure 1 can be replaced by two oscillators which are phase locked or injection locked as long as they have same amplifier gain.

A. Implementation of signal separation

The principle is that input signal is split into envelope component and constant envelope angle-modulated component which also called baseband component and carrier component. Then the former is modulated in the phase of the latter in one branch and phase -inversely modulated in the other branch. After up conversion and amplified, the two branches are subtracted and the envelope 
component is demodulated from phase. As a result, the input signal is restored and amplified.

As is shown in Figure 2, the purpose of envelope elimination circuit is to remove envelope from input signal and obtain carrier component. After passing through envelope remove circuit, $S(t)$, the input band-pass signal, is transformed into constant envelope angle-modulated signal $P(t)$, then the envelope signal $E^{\prime}(t)$ is achieved from either synchronous detector or a linear envelope detector. And

$$
\begin{gathered}
P(t)=K \cos \left[\omega_{0} t+\theta(t)\right] \\
E(t)=E_{m} \sin \phi(t) \\
E^{\prime}(t)=\frac{K}{2} E(t)
\end{gathered}
$$
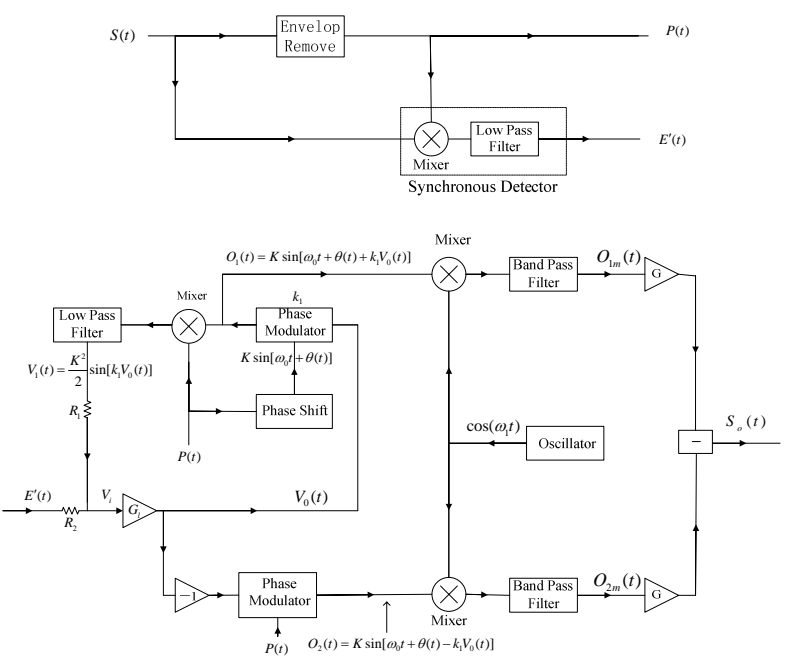

Figure 2. System block of LINC amplifier.

To obtain two separate signals, amplifier's feedback loop is analyzed firstly. The amplifier has a very large voltage gain $G_{l}=-V_{0} / V_{i}$. A phase-shifted signal of $P(t)$ is modulated by $V_{0}(t)$, after passing through mixer and low pass filter, the production is

$$
V_{1}(t)=\frac{K^{2}}{2} \sin \left[k_{1} V_{0}(t)\right]
$$

For closed loop stability let $\left|k_{1} V_{0}(t)\right| \leq \pi / 2$, and assume that the amplifier input impedance is large enough compared to $R_{1}$ and $R_{2}$, so that

$$
V_{0}(t)\left[1+G_{l} \frac{\left(K^{2} / 2\right) R_{2} \sin k_{1} V_{0}(t)}{\left(R_{1}+R_{2}\right) V_{0}(t)}\right]=-G_{l} \frac{E^{\prime}(t) R_{1}}{R_{1}+R_{2}}
$$

For $\left|k_{1} V_{0}(t)\right| \leq \pi / 2, \frac{\sin k_{1} V_{0}(t)}{V_{0}(t)}$ has a minimum of $2 k_{1} / \pi$, and it has a maximum of $k_{1}$ when $V_{0}$ is small and $\sin k_{1} V_{0}(t) \approx k_{1} V_{0}(t) \quad$. When $\quad G_{l} \square\left[\frac{\left(R_{1}+R_{2}\right)}{K^{2} R_{2}}\right]\left(\frac{\pi}{k_{1}}\right)$,

equation (7) becomes

$$
E(t) \approx-K \frac{R_{2}}{R_{1}} \sin k_{1} V_{0}(t)
$$

It is different with the formula in reference[6] , $E(t) \approx-\frac{K^{2}}{2} \frac{R_{2}}{R_{1}} \sin k_{1} V_{0}(t)$. The reason is that the signal after the synchronous detector is $E^{\prime}(t)$ other than $E(t)$.

It is obvious that (8) has the same form as (4) provided $G_{l}$ is large enough and the approximation can be made. The size of $G_{l}$ will be determined by the distortion limits placed on the overall LINC amplifier. If $k_{1}$ and $K$ are sufficiently large, $G_{l}$ could be less than unity gain and the baseband amplifier could be replaced by a passive addition network, which could increase the realizable bandwidth of component separator. Assume that the values of $K, R_{1}$ and $R_{2}$ are chosen to satisfy that $K \cdot R_{2} / R_{1}=E_{m}$, then

$$
k_{1} V_{0}(t)=-\phi(t)
$$

and then the output in Figure 2 becomes

$$
\begin{aligned}
& O_{1}(t)=K \sin \left[\omega_{0} t+\theta(t)-\phi(t)\right]=\left(2 K / E_{m}\right) S_{2}(t) \\
& O_{2}(t)=K \sin \left[\omega_{0} t+\theta(t)+\phi(t)\right]=\left(2 K / E_{m}\right) S_{1}(t)
\end{aligned}
$$

Of course the feedback loops should be designed to meet the requirement of phase-shift and gain stability. Note that if the phase modulator in feedback loop cannot produce a linear phase change as a function of modulating voltage $V_{0}$ (such as $k_{1}$ is a function of $V_{0}$ ), then the high-gain feedback loop will compensate that by distorted $V_{0}(t)$ so that $\sin \left[k_{1}\left(V_{0}\right) V_{0}(t)\right]=V_{1}(t)$ and $k_{1}\left(V_{0}\right) V_{0}(t)=\phi(t)$. That is the only requirement the two phase modulators must have the same modulation characteristic $k_{1}\left(V_{0}\right)$. If the modulator with a characteristic $\sin ^{-1}$ could be realized, the feedback loop would not be required.

\section{B. Frequency conversion and amplification}

The low-frequency signal is separated into components $O_{1}(t)$ and $O_{2}(t)$ which are translated in frequency with the same mixing oscillator, and then amplified as is shown in Figure 2 to produce

$$
\begin{aligned}
& O_{1 m}(t)=\frac{K}{2} \sin \left[\left(\omega_{0}+\omega_{1}\right) t+\theta(t)-\phi(t)\right] \\
& O_{2 m}(t)=\frac{K}{2} \sin \left[\left(\omega_{0}+\omega_{1}\right) t+\theta(t)+\phi(t)\right]
\end{aligned}
$$

The mixers and amplifiers can be nonlinear. The output is the amplified signal whose frequency is converted into $\omega_{0}+\omega_{1}$. At last, through a subtractor the signal becomes 
$S_{o}(t)=G O_{2 m}-G O_{1 m}=\frac{K G}{E_{m}} E(t) \cos \left[\left(\omega_{0}+\omega_{1}\right) t+\theta(t)\right](12)$

It is with a constant of $\frac{K G}{E_{m}}$ other than $\frac{4 K G}{E_{m}}$ in reference[6], it is concluded that LINC amplifier realizes $G$ gain as indicated in Figure 1 only when $K=E_{m}$.

\section{SimULATION AND ANALYSIS}

Assume $R_{1}=100 \Omega, R_{2}=200 \Omega$, the input baseband signal is with $\theta(t)=1000 t, \phi(t)=2 \pi \times 10^{5} t$. The outputs of the circuit are simulated for the changes of carrier frequency, baseband frequency, input amplitude and $K$.

\section{A. For $320 \mathrm{MHz}$ carrier, baseband at $50 \mathrm{MHz}$ and $\mathrm{K}=0.5$}

When the carrier frequency is $f_{1}=\omega_{1} / 2 \pi=320 \mathrm{MHz}$, the frequency of input constant envelope component is $f_{0}=\omega_{0} / 2 \pi=50 \mathrm{MHz}$, the simulated results of LINC amplifier is shown in Figure 3, where amplitudes of input and output are $1 \mathrm{~V}$ and $5 \mathrm{~V}$ respectively, voltage gain is 5 times and power are concentrated in $49.9 \mathrm{MHz}$ and 369.3MHz.
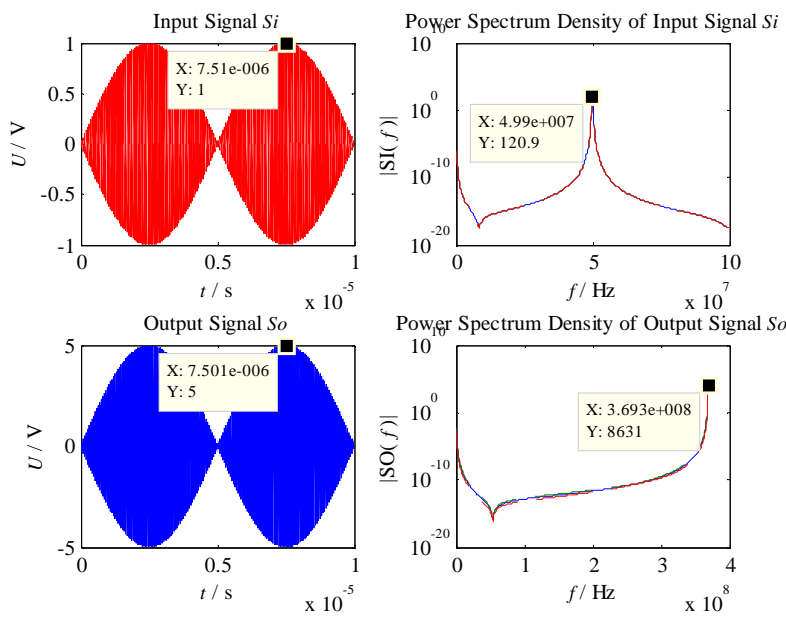

Figure 3. Result of program simulation.

\section{B. For $320 \mathrm{MHz}$ carrier, baseband at $5 \mathrm{MHz}$ and $\mathrm{K}=0.5$}

When frequency of input constant envelope component is changed into $f_{0}=5 \mathrm{MHz}$ and carrier frequency stay unchanged, with properly adjusting sampling frequency Figure 4 is obtained. In the figure, the amplitude of output voltage is unchanged and there is no distortion in amplification. As for the two peaks in power spectrum density, it is a result of two- tone signal.
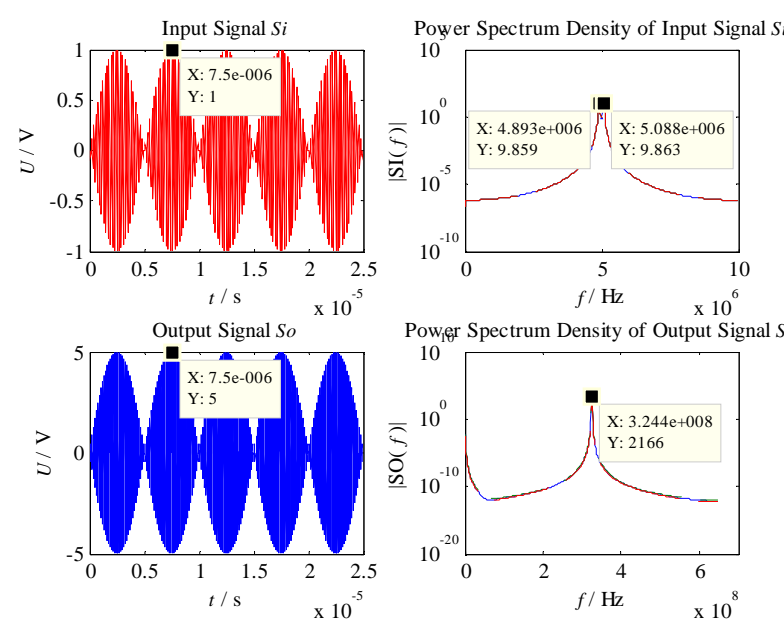

Figure 4. Change of baseband frequency and its simulation diagram

Input signal $S(t)=E_{m} \sin (\phi(t)) \cdot \cos \left(\omega_{0} t+\theta(t)\right)$ can be treated as two-tone signal which has two frequencies $f_{0}+\frac{\theta}{2 \pi t}+\frac{\phi}{2 \pi t}$ and $f_{0}+\frac{\theta}{2 \pi t}-\frac{\phi}{2 \pi t}$, and so can output signal. It is determined by the ratio of $f_{0}$ to $\frac{\phi}{2 \pi t}$ whether there is a visible spectral diffusion in the figure or not.

\section{For $3 \mathrm{GHz}$ carrier, baseband at $50 \mathrm{MHz}$ and $\mathrm{K}=0.5$}

When baseband frequency stays at $50 \mathrm{MHz}$ and carrier frequency is replaced by $3 \mathrm{GHz}$, Figure 5 is produced, where amplitude of output signal has no change and is amplified without distortion.
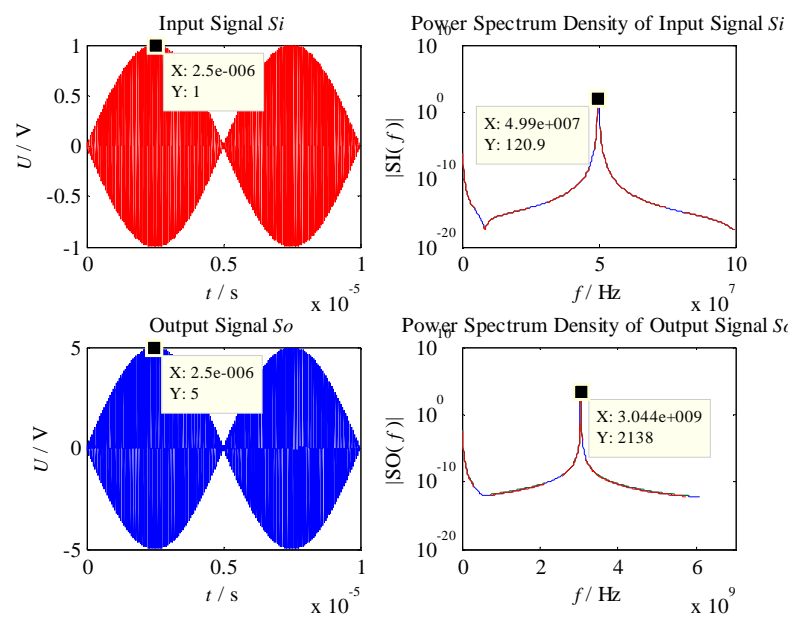

Figure 5. Change of carrier frequency and its simulation diagram.

\section{For $10 \mathrm{~V}$ signals}

From the foregoing, LINC amplifier operates without distortion under different baseband frequency and carrier frequency. 
When amplitude of input signal is changed into $10 \mathrm{~V}$ and coefficient of envelope elimination circuit $K$ is changed into 5 , the result is shown in Figure 6. It follows that LINC amplifier works without distortion under different amplitude of input signal.
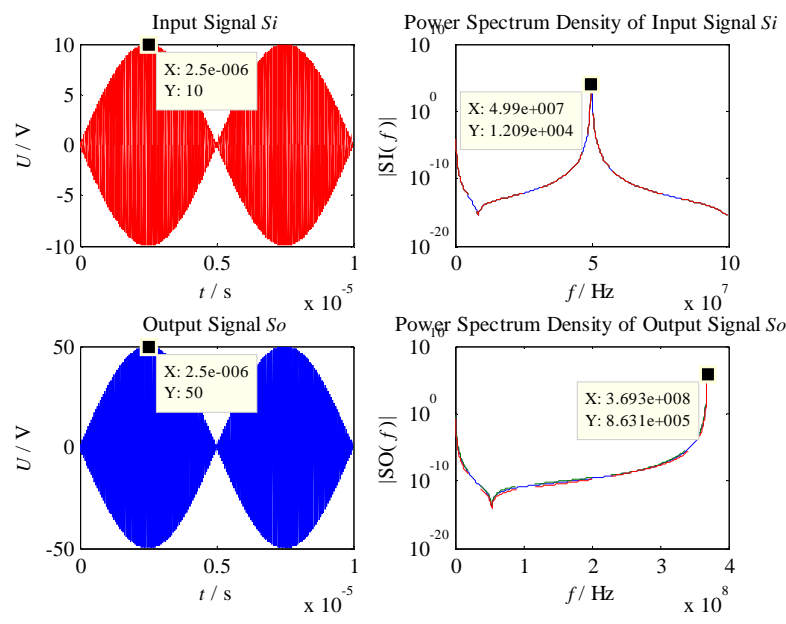

Figure 6. Change of amplitude and its simulation diagram.

\section{CONCLUSIONS}

LINC technique is applied to non-constant amplitude and phase signal. It realizes linear amplification by means of transforming input signal into constant envelope signal. The amplifier is able to work in nonlinear region (saturation region), that's why it has high efficiency and processes signal of large PAPR without distortion. However, there must be a precise balance between the two branches and no phase delay or amplitude deviation, and two amplifiers must have the same characteristic absolutely. Here an improvement is brought up. With the research on COX's theory, improvement is made, simulation and its analysis is carried on. The result shows that LINC technique is suitable for different amplitude and frequency, and amplification factor is codetermined under the condition without loss by amplitude of envelope component, coefficient of envelope elimination circuit and amplifier gain. The value is $\frac{K G}{E_{m}}$.

\section{ACKNOWLEDGEMENT}

This work is supported by National Natural Foundation of China (61172035) and China Postdoctoral Science Foundation (20110491875).

\section{REFERENCES}

[1] S. C. Cripps, RF power amplifier design for wireless communication. 2nd ed. Boston: Artech House, 2006.

[2] S. A. Hetzel, A. Bateman, and J. P. Mcgeehan, "A LINC transmitter," Vehicular Technology Conference, Gateway to the Future Technology in Motion., 41st IEEE, 1991, pp. 133- 137.

[3] Mao Wen-jie, "RF Power Amplifier Linearity Study based on Predistortion Technology,” Hangzhou: Zhejiang University, 2005.

[4] A. Birafane, M. El-Asmar, A. B. Kouki, M. Helaoui, and F. M. Ghannouchi, “Analyzing LINC system,” Microwave Magazine, IEEE, 2010, vol. 11, pp. 59-71.

[5] P. Colantonio, F. Giannini, and M. Rossi, “A RF approach for the implementation of the LINC technique," Proceedings of the 36th European Microwave Conference, Italy, 2006, pp. 1143-1146.

[6] D. C. Cox, "Linear amplification with nonlinear components," IEEE Transactions on Communications, 1974, vol. 23, pp. 1942-1945.

[7] D. C. Cox and R. P. Leck, "Component signal separation and recombination for linear amplification with nonlinear components," IEEE Transactions on Communications, 1975, vol. 23, pp. 1281-1287.

[8] B. Shi and M. Y. W. Chia, "A power efficient companding LINC architecture," Proceedings of the 40th European Microwave Conference, Paris, 2010, pp. 1010-1013.

[9] M. Helaoui, S. Boumaiza, A. Ghazel, and F. M. Ghannouchi, "Digital compensation of branches imbalance effects in LINC transmitters," The 16th International Conference on Microelectronics, 2004, pp. 688-691.

[10] Y. C. Chen, K. Y. Jheng, A. Y. Wu, H. W. Tsao, and B. Tzeng, "Multilevel LINC system design for wireless transmitters," International Symposium on VLSI Design, Automation and Test, 2007, pp. 1-4.

[11] T. W. Chen, P. Y. Tsai, J. Y. Yu, and C. Y. Lee, "A low-power alldigital signal component separator for OFDM LINC systems," International Conference on Green Circuits and Systems (ICGCS), 2010, pp. 328-333. 